OPEN ACCESS

Edited by:

Ying Han,

Capital Medical University, China

Reviewed by:

Parnetti Lucilla,

University of Perugia, Italy

Tong $L i$,

Johns Hopkins University,

United States

*Correspondence:

Gerard Piñol-Ripoll

gerard_437302@hotmail.com

${ }^{\dagger}$ These authors have contributed equally to this work and share first

authorship

Received: 08 September 2020

Accepted: 10 February 2021

Published: 04 March 2021

Citation:

Dakterzada F, López-Ortega $R$, Arias A, Riba-Llena I, Ruiz-Julián M, Huerto R, Tahan N and Piñol-Ripoll G (2021) Assessment of the

Concordance and Diagnostic

Accuracy Between Elecsys and Lumipulse Fully Automated Platforms and Innotest.

Front. Aging Neurosci. 13:604119. doi: 10.3389/fnagi.2021.604119

\section{Assessment of the Concordance and Diagnostic Accuracy Between Elecsys and Lumipulse Fully Automated Platforms and Innotest}

\author{
Farida Dakterzada ${ }^{\dagger}$, Ricard López-Ortega ${ }^{\dagger}$, Alfonso Arias ${ }^{\dagger}$, Iolanda Riba-Llena', \\ Maria Ruiz-Julián ${ }^{\dagger}$, Raquel Huerto ${ }^{\dagger}$ Nuria Tahan' ${ }^{\dagger}$ and Gerard Piñol-Ripoll* \\ Cognitive Disorders Unit, Clinical Neuroscience Research Group, Santa Maria University Hospital, IRBLleida, Lleida, Spain
}

Manual ELISA assays are the most commonly used methods for quantification of biomarkers; however, they often show inter- and intra-laboratory variability that limits their wide use. Here, we compared the Innotest ELISA method with two fully automated platforms (Lumipulse and Elecsys) to determine whether these new methods can provide effective substitutes for ELISA assays. We included 149 patients with AD $(n=34), \mathrm{MCl}(n=94)$ and non-AD dementias $(n=21)$. A $(n 42$, T-tau, and P-tau were quantified using the ELISA method (Innotest, Fujirebio Europe), CLEIA method on a Lumipulse G600II (Fujirebio Diagnostics), and ECLIA method on a Cobas e 601 (Roche Diagnostics) instrument. We found a high correlation between the three methods, although there were systematic differences between biomarker values measured by each method. Both Lumipulse and Elecsys methods were highly concordant with clinical diagnoses, and the combination of Lumipulse A $\beta 42$ and P-tau had the highest discriminating power (AUC 0.915, 95\% Cl 0.822-1.000). We also assessed the agreement of $A T(N)$ classification for each method with $A D$ diagnosis. Although differences were not significant, the use of $A \beta 42 / A \beta 40$ ratio instead of $A \beta 42$ alone in AT(N) classification enhanced the diagnostic accuracy (AUC $0.798,95 \% \mathrm{Cl} 0.649-$ 0.947 vs. AUC $0.778,95 \% \mathrm{Cl} 0.617-0.939$ ). We determined the cut-offs for the Lumipulse and Elecsys assays based on the $A \beta 42 / A \beta 40$ ratio \pm status as a marker of amyloid pathology, and these cut-offs were consistent with those recommended by manufacturers, which had been determined based on visual amyloid PET imaging or diagnostic accuracy. Finally, the biomarker ratios (P-tau/A $\beta 42$ and T-tau/A $\beta 42)$ were more consistent with the $A \beta 42 / A \beta 40$ ratio for both Lumipulse and Elecsys methods, and Elecsys P-tau/A $\beta 42$ had the highest consistency with amyloid pathology (AUC 0.994, 95\% Cl 0.986-1.000 and OPA 96.4\%) at the $\geq 0.024$ cut-off. The Lumipulse and Elecsys cerebrospinal fluid (CSF) AD assays showed high analytical and clinical performances. As both automated platforms were standardized for reference samples, their use is recommended for the measurement of CSF AD biomarkers compared with unstandardized manual methods, such as Innotest ELISA, that have demonstrated a high inter and intra-laboratory variability.

\footnotetext{
Keywords: Alzheimer's disease, assay automation, biomarker, cerebrospinal fluid, cut-off
} 


\section{INTRODUCTION}

Alzheimer's disease $(\mathrm{AD})$ is the most prevalent age-related neurodegenerative disease, accounting for $60-80 \%$ of cases of dementia. The extracellular amyloid plaques arising from the accumulation of amyloid $\beta 42$ protein $(\mathrm{A} \beta 42)$ and intracellular neurofibrillary tangles formed by aggregations of hyperphosphorylated tau protein (P-tau) are the two main pathological hallmarks of AD (Serrano-Pozo et al., 2011). Both of these pathological characteristics are specific to $A D$, while neurodegeneration, characterized by an increase in total-tau protein (T-tau), is a non-specific biomarker that can be caused by several neurodegenerative diseases (Jack et al., 2018). A $\beta 42$, $\mathrm{P}$-tau, and T-tau are considered core AD biomarkers that can be measured in cerebrospinal fluid (CSF). Their use increases the accuracy of the diagnosis and prediction of the progression from mild cognitive impairment (MCI) to $\mathrm{AD}$ and can differentiate between $\mathrm{AD}$ and other causes of dementia or neuropsychiatric problems (Albert et al., 2011; McKhann et al., 2011; Sperling et al., 2011). In addition, the inclusion of these biomarkers in diagnosis benefits populations included in clinical trials (Jack et al., 2018).

Currently, enzyme-linked immunoassay (ELISA) is the most widely used approach for the detection of AD core biomarkers in CSF. However, these ELISA methods often show considerable inter and intra-lab variability that prevents the use of standard cut-off values and precludes the wide use of CSF biomarkers in clinical practice. To circumvent this problem, Fujirebio Diagnostics and Roche Diagnostics have recently developed fully automated platforms for the analysis of CSF biomarkers. Fujirebio has implemented four CSF analytes $(\mathrm{A} \beta 42, \mathrm{~A} \beta 40$, T-tau, and P-tau) on the fully automated Lumipulse G System. The measurement method is based on a two-step sandwich chemiluminescent enzyme-immunoassay (CLEIA). The Lumipulse $\mathrm{A} \beta 42$ assay is standardized according to certified reference material (CRM) developed by the International Federation of Clinical Chemistry and Laboratory Medicine (IFCC) and the Joint Research Centre (JRC). These platforms consist of three CRMs based on human CSF, with low, middle and high concentrations of $A \beta 42$. However, fully automated Elecsys assays for CSF A $\beta 42$, T-tau and P-tau are run on Elecsys and Cobas e immunoassay analyzers (Roche Diagnostics $\mathrm{GmbH}$, Penzberg, Germany). The measurement is performed based on the electrochemiluminescence immunoassay (ECLIA) in a two-step sandwich assay. The Elecsys $\mathrm{A} \beta 42$ assay has been standardized by a Joint Committee for Traceability in Laboratory Medicine (JCTLM) with an approved reference measurement procedure (RMP). Therefore, all assay lots are standardized to a sample set with target values derived from liquid chromatography-tandem mass spectrometry (LC-MS/MS) (Leinenbach et al., 2014).

Previous studies have evaluated the consistency between each of these automated methods with manual ELISA methods or Amyloid PET imaging (Janelidze et al., 2017; Hansson et al., 2018; Kollhoff et al., 2018; Schindler et al., 2018; Willemse et al., 2018; Alcolea et al., 2019; Bayart et al., 2019; Zecca et al., 2019; Kaplow et al., 2020). However, there are no studies that have compared the efficacy of Innotest, Lumipulse and Elecsys methods in a single cohort of patients.

The aims of this study were (a) to assess the concordance between core AD biomarkers measured in CSF using Innotest, Lumipulse and Elecsys methods; (b) to evaluate the diagnostic accuracy of biomarkers and their ratios measured by each method; (c) to assess the discriminating power of $\mathrm{AT}(\mathrm{N})$ groups that were generated by the results of the different biomarkers for each of these three technologies and (d) to define the CSF cutoff points for both Lumipulse and Elecsys assays based on the Lumipulse $A \beta 42 / 40$ status.

\section{MATERIALS AND METHODS}

\section{Study Population}

A total of 149 patients [AD $(n=34)$, MCI $(n=94)$ and non-AD dementias $(n=21)]$ were included in this study. The study population was recruited consecutively between July 2018 and July 2019 from patients attending the Cognitive Disorders Unit at the Hospital Universitari Santa Maria (Lleida, Spain). Inclusion criteria comprised presentation with suspected cognitive dysfunction at the memory clinic, for which the neurologist requested CSF analysis. The diagnosis of probable $\mathrm{AD}$ and $\mathrm{MCI}$ was performed based on NIAA criteria (Albert et al., 2011; McKhann et al., 2011). Each non-AD patient fulfilled the criteria for the specific diagnostic criteria of the disorder considered (e.g., Fronto-temporal dementia, Lewy body dementia, etc.) (Gorno-Tempini et al., 2011; Rascovsky et al., 2011; McKeith et al., 2017). The included patients signed an internal regulatory document stating that residual samples used for diagnostic procedures could be used for research studies without any additional informed consent.

\section{CSF Collection and Storage}

Cerebrospinal fluid samples were collected between 8 a.m. and 10 a.m. after an overnight fast into $10-\mathrm{mL}$ polypropylene tubes (Sarstedt, 62.610.201). The tubes were inverted several times, and the CSF was processed based on the recommendations provided by each manufacturer. For the Lumipulse assay, the samples were centrifuged at $2,000 \times g$ for $10 \mathrm{~min}$ at room temperature and aliquoted into two 2 -mL polypropylene tubes (Sarstedt, 72.694.007), with each tube containing $1 \mathrm{~mL}$ of CSF. For the Elecsys method, the samples were aliquoted into two $0.5-\mathrm{mL}$ polypropylene tubes (Sarstedt 72.730.005) after centrifugation. For the Innotest assay, the CSF was aliquoted into two $2-\mathrm{mL}$ polypropylene tubes (Sarstedt, 72.694.007) after centrifugation. The samples were stored at $-80^{\circ} \mathrm{C}$ until analyses.

\section{CSF Analysis}

Measurements of $A \beta 42$ and $A \beta 40$ (only for lumipulse), T-tau, and P-tau were performed at the clinical laboratory of Hospital Universitari Arnau de Vilanova, Lleida. On the day of the analysis, samples were thawed at room temperature, and the tubes were vortexed briefly. The biomarkers were measured directly from the storage tube and in five separate batches for all three methods. For each method, the same batch of reagents 
was used for each biomarker throughout the study, and for each sample, all analytes were quantified in the same run and from the same aliquot. For the ELISA method, Innotest A $\beta 42$, Innotest htau-Ag, and Innotest P-tau (181P) assays (Fujirebio, Europe) were used. Innotest calibrator concentrations ranged from 63 to $4000 \mathrm{pg} / \mathrm{mL}$ for $\mathrm{A} \beta 42,40$ to $2300 \mathrm{pg} / \mathrm{mL}$ for T-tau, and 16 to $1000 \mathrm{pg} / \mathrm{mL}$ for P-tau. According to previous analyses in clinical practice, cut-offs at our center were determined to be $<600 \mathrm{pg} / \mathrm{mL}$ for $\mathrm{A} \beta 42,>425 \mathrm{pg} / \mathrm{mL}$ for T-tau, and $>65 \mathrm{pg} / \mathrm{mL}$ for P-tau. For the ECLIA method, the tubes analyzed using the Elecsys A $\beta 42$ CSF, Elecsys T-tau CSF, and Elecsys P-tau (181P) CSF assays (Roche Diagnostics $\mathrm{GmbH}$ ) were run on the cobas e 601 analyzer (Roche Diagnostics) per the manufacturer's instructions. Elecsys measuring ranges were as follows: 200 to $1700 \mathrm{pg} / \mathrm{mL}$ for $\mathrm{A} \beta 42,80$ to $1300 \mathrm{pg} / \mathrm{mL}$ for T-tau, and 8 to $120 \mathrm{pg} / \mathrm{mL}$ for P-tau. For data analysis, we used the cut-offs recommended by the manufacturer, which were as follows: $\leq 1000 \mathrm{pg} / \mathrm{mL}$ for $\mathrm{A} \beta 42,>300 \mathrm{pg} / \mathrm{mL}$ for T-tau, and $>27 \mathrm{pg} / \mathrm{mL}$ for P-tau. Seventeen samples had $\mathrm{A} \beta 42$ levels above the upper limit of the measuring range $(1700 \mathrm{pg} / \mathrm{mL})$ and were eliminated from the analysis. The results of the Elecsys $\mathrm{A} \beta 42$ assay were standardized to the JCTLM-approved RMP for quantitation of $A \beta 42$ in human CSF, based on LC-MS/MS (Leinenbach et al., 2014). For the CLEIA technology, the CSF biomarkers were quantified using the Lumipulse $A \beta 42, A \beta 40$, T-tau, and P-tau (181P) assays on the LUMIPULSE G600II automated platform (Fujirebio) per the manufacturer's instructions. Lumipulse measuring ranges were 9-2,335 $\mathrm{pg} / \mathrm{mL}$ for $\mathrm{A} \beta 42,150-2,000 \mathrm{pg} / \mathrm{mL}$ for T-tau, and $1.1-400 \mathrm{pg} / \mathrm{mL}$ for $\mathrm{p}$-Tau. The following cut-offs that had been determined by Fujirebio were used for data analysis: $\mathrm{A} \beta 42<600 \mathrm{pg} / \mathrm{mL}, \mathrm{A} \beta 42 / 40<0.069$, T-tau > $400 \mathrm{pg} / \mathrm{mL}$, and P-tau $>56.5 \mathrm{pg} / \mathrm{mL}$. The results of the Lumipulse A $\beta 42$ presented in this work have been standardized with CRMs developed by the IFCC and JRC (Kuhlmann et al., 2017). The personnel involved in the CSF analyses were blind to the clinical diagnosis.

\section{Statistical Analyses}

All statistical analyses were performed using IBM SPSS version 25 (Armonk, NY, United States). One-way ANOVA and Chi-square tests were used for analysis of quantitative and qualitative variables, respectively. The quantitative variables were presented as means ( \pm standard deviation, SD), and the qualitative variables were presented as percentages. To evaluate the correlation between methods, we used Pearson's correlation coefficient ( $\mathrm{r}$ ), paired $t$-tests for paired samples, and the Bland-Altman plot. The diagnostic accuracy of the biomarkers/AT(N) classification was analyzed using a binary logistic regression model. In this model, the sensitivity was defined as the percentage of correct classification of $\mathrm{AD}$ diagnosis and the specificity as the percentage of correct classification of non-AD dementias diagnosis. Furthermore, we used this statistical model to evaluate the predictive value of the biomarkers with respect to $\mathrm{AD}$ prognosis. The receiver operating characteristic (ROC) analysis for evaluating diagnostic accuracy was further analyzed using the Hanley and McNeil method (Hanley and McNeil, 1982) to compare the Area Under the Curve (AUC). Values of $|z| \geq 1.96$ were considered evidence that the true ROC areas were different. We also performed ROC analysis to determine the cut-offs for the core $\mathrm{AD}$ biomarkers and the ratios that best distinguished Lumipulse $A \beta 42 / 40+$ individuals. In addition, the cut-offs were also determined based on the Innotest $A \beta 42$ status. We determined the positive percent agreement (PPA) and negative percent agreement (NPA), and the single analyte value (or ratio) with the highest Youden index (PPA + NPA - 1) was identified as the cut-off value. Overall percent agreement (OPA) was defined as the sum of the $A \beta 42 / 40+$ individuals who were positive for a CSF biomarker measure and the A $342 / 40$ - individuals who were negative for a CSF biomarker measure divided by the cohort size, thereby providing an estimate of accuracy.

\section{RESULTS}

\section{Patient Characteristics}

The demographic characteristics and biomarker results are summarized in Table 1 . The average age of participants was 74 years, and 55\% were female. Syndrome diagnoses in the cohort were the following: $34(22.8 \%)$ with $\mathrm{AD}, 94$ (63.1\%) with MCI, and 21 (14.1\%) with non-AD dementia. There were no significant differences between diagnostic groups for demographic and clinical variables except for MMSE score and hypertension $(P<0.0001$ and $P<0.05$, respectively). The mean MMSE score was lower [19.6 (4.2 $\mathrm{SD})$ ] for $\mathrm{AD}$ patients compared with the two other groups, followed by non-AD dementia patients [21.9 (4.6 SD)] and MCI subjects [25.2 (3.1 SD)]. For all three assays, all CSF biomarker concentrations were significantly different between the three diagnostic groups, except Lumipulse A $\beta 40$ $(P>0.05)$. For Elecsys, samples that had $A \beta 42$ values above the upper limit of detection $(1700 \mathrm{pg} / \mathrm{mL})$ were omitted from analysis ( $n=17$ samples, MCI 11, AD 4, and 2 non$\mathrm{AD}$ patients).

\section{Concordance Between Innotest and Lumipulse Assays}

Pearson's correlations indicated a high correlation between biomarkers of both methods. Figure 1 shows the correlation and Bland-Altman plots for biomarkers quantified by Innotest and Lumipulse. The correlation coefficient between the two methods was 0.87 for $\mathrm{A} \beta 42(P<0.0001), 0.95$ for $\mathrm{T}$-tau $(P<0.0001)$ and 0.95 for P-tau $(P<0.0001)$. The concordance between the values of the biomarkers between the two methods was assessed using paired sample $t$-tests. Our results indicated that there was high consistency in the $\mathrm{A} \beta 42$ (observed slope $0.98, t$-test $p=0.319$ ) and T-tau (observed slope $0.96, t$-test $P=0.785$ ) values between the two methods (Figure 1). Lumipulse A $\beta 42$ values were slightly higher than those for Innotest, while Lumipulse T-tau values were slightly lower than those for Innotest; however, these differences were not statistically significant (Figure 1). As shown in the Bland-Altman plot, the bias (mean of the differences) for $\mathrm{A} \beta 42$ 
TABLE 1 | The demographic characteristics and biomarker results for $A D, M C l$, and non-AD patients.

\begin{tabular}{|c|c|c|c|c|c|}
\hline & All participants & $A D$ & $\mathrm{MCl}$ & Non-AD dementia & $P$-value \\
\hline$n(\%)$ & $149(100 \%)$ & $34(22.8 \%)$ & $94(63.1 \%)$ & $21(14.1 \%)$ & \\
\hline Age (years) & $73.82(6.85)$ & $74.00(8.78)$ & $73.86(6.05)$ & $73.33(7.07)$ & 0.937 \\
\hline Sex (\% female) & $55.7 \%$ & $67.6 \%$ & $54.3 \%$ & $42.9 \%$ & 0.178 \\
\hline MMSE score & $23.41(4.29)$ & $19.62(4.24)$ & $25.16(3.07)$ & $21.90(4.57)$ & $<0.0001$ \\
\hline Family history of cognitive impairment & $28.9 \%$ & $23.5 \%$ & $29.8 \%$ & $33.3 \%$ & 0.7 \\
\hline Hypertension & $57.7 \%$ & $67.6 \%$ & $55.3 \%$ & $52.4 \%$ & 0.003 \\
\hline Diabetes Mellitus & $20.1 \%$ & $26.5 \%$ & $19.1 \%$ & $14.3 \%$ & 0.509 \\
\hline Dyslipidemia & $44.3 \%$ & $47.1 \%$ & $44.7 \%$ & $38.1 \%$ & 0.803 \\
\hline Depression & $35.6 \%$ & $29.4 \%$ & $36.2 \%$ & $42.9 \%$ & 0.587 \\
\hline \multicolumn{6}{|l|}{ Innotest } \\
\hline $\mathrm{A} \beta 42 \mathrm{pg} / \mathrm{mL}$ & $581.37(247.46)$ & 405.65 (115.36) & $614.11(252.18)$ & 722.48 (239.53) & $<0.0001$ \\
\hline T-tau pg/mL & 507.85 (354.32) & 684.47 (393.01) & $450.71(277.00)$ & 477.67 (498.05) & 0.003 \\
\hline P-tau pg/mL & 67.55 (28.62) & $83.81(34.75)$ & $64.68(25.10)$ & $53.91(21.14)$ & $<0.0001$ \\
\hline T-tau/A $\beta 42$ & $1.130(1.092)$ & 1.908 (1.623) & $0.935(0.743)$ & $0.727(0.687)$ & $<0.0001$ \\
\hline P-tau/A $\beta 42$ & $0.148(0.116)$ & $0.234(0.167)$ & $0.130(0.083)$ & $0.088(0.056)$ & $<0.0001$ \\
\hline \multicolumn{6}{|l|}{ Elecsys } \\
\hline $\mathrm{A} \beta 42 \mathrm{pg} / \mathrm{mL}$ & 770.69 (363.12) & $572.50(179.97)$ & 807.75 (369.95) & 970.04 (433.81) & $<0.0001$ \\
\hline T-tau pg/mL & $287.80(155.38)$ & 379.65 (188.44) & 261.39 (118.65) & 251.37 (189.67) & $<0.0001$ \\
\hline P-tau pg/mL & $27.38(17.17)$ & 38.92 (23.19) & $24.73(13.22)$ & $19.56(11.71)$ & $<0.0001$ \\
\hline T-tau/A $\beta 42$ & $0.463(0.338)$ & $0.719(0.470)$ & $0.390(0.225)$ & $0.318(0.242)$ & $<0.0001$ \\
\hline P-tau/A $\beta 42$ & $0.045(0.040)$ & $0.075(0.060)$ & $0.038(0.024)$ & $0.027(0.022)$ & $<0.0001$ \\
\hline \multicolumn{6}{|l|}{ Lumipulse } \\
\hline $\mathrm{A} \beta 42 \mathrm{pg} / \mathrm{mL}$ & $571.43(276.75)$ & 415.28 (119.15) & 599.55 (289.63) & 698.41 (301.96) & $<0.0001$ \\
\hline $\mathrm{A} \beta 40 \mathrm{pg} / \mathrm{mL}$ & 10317.68 (3339.78) & 10597.44 (3605.39) & 10363.14 (3111.28) & 9661.24 (3935.09) & 0.59 \\
\hline $\mathrm{A} \beta 42 / 40$ & $0.056(0.022)$ & $0.041(0.010)$ & $0.058(0.022)$ & $0.073(0.022)$ & $<0.0001$ \\
\hline T-tau pg/mL & 510.37 (356.34) & 731.85 (404.16) & $438.84(256.48)$ & $471.95(505.21)$ & $<0.0001$ \\
\hline P-tau pg/mL & $81.50(56.72)$ & $122.96(73.80)$ & $72.76(44.18)$ & $53.49(40.49)$ & $<0.0001$ \\
\hline T-tau/A $\beta 42$ & $1.144(1.037)$ & $1.933(1.504)$ & $0.940(0.700)$ & $0.779(0.740)$ & $<0.0001$ \\
\hline P-tau/A $\beta 42$ & $0.190(0.190)$ & $0.330(0.291)$ & $0.159(0.123)$ & $0.101(0.101)$ & $<0.0001$ \\
\hline
\end{tabular}

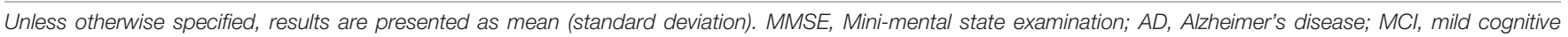

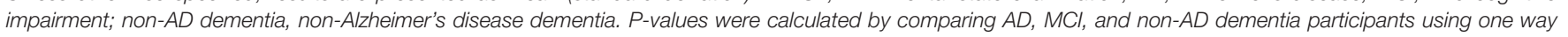
ANOVA for continuous variables and Pearson Chi2 for categorical variables.

was 11.320 units $(\mathrm{pg} / \mathrm{mL})$ (continuous line) between the two methods. The regression line for the differences indicated that there was a non-significant negative trend in the differences as the magnitude of the measured variable increased. For T-tau, there was a bias of -2.157 units between the two methods. However, the results of Lumipulse and Innotest were not consistent with respect to $\mathrm{P}$-tau values (observed slope 1.884, $t$-test $P<0.0001$ ). For P-tau, there was a bias of -14.328 units (continuous line). The regression line for the differences indicated that there was a systematic proportional bias between the values of the two methods with a negative trend in the differences as the magnitude of P-tau values increased, especially for values greater than 50 $\mathrm{pg} / \mathrm{mL}$. Among all assays evaluated, approximately $95 \%$ of the measured values were within $\pm 1.96 \mathrm{SD}$ of the bias (Figure 1).

\section{Concordance Between Innotest and Elecsys Assays}

Pearson's correlations indicated a high correlation between biomarkers of both methods. The correlation coefficient between the two methods was 0.88 for A $\beta 42(P<0.0001), 0.96$ for T-tau $(P<0.0001)$ and 0.97 for P-tau $(P<0.0001)$. The paired samples $t$-test demonstrated that there was weak concordance between the two methods for all of the biomarkers. For all three biomarkers, the adjustment line (continuous line) (observed slope of 0.52 for $\mathrm{A} \beta 42$, $t$-test $P<0.0001$; observed slope of 2.05 for T-tau, $t$-test $P<0.0001$; and observed slope of 1.62 for P-tau, $t$-test $P<0.0001)$ was significantly separated from the perfect agreement line (dashed line) (Figure 2). The Bland-Altman plot indicated that there was a bias of -222.13 units (continuous line) between the two methods for $A \beta 42$ (i.e., the Elecsys method quantified on average $222.13 \mathrm{pg} / \mathrm{mL}$ more $\mathrm{A} \beta 42$ than the Innotest assay). The regression line demonstrated a proportional systematic bias with a negative trend of differences as the magnitude of $\mathrm{A} \beta 42$ increased. For T-tau and P-tau, the biases (mean of differences) were 210.70 and 40.16 units, respectively. The regression line of the differences indicated a proportional systematic bias for both biomarkers with a positive trend of differences as the magnitude of these biomarkers increased. For all assays 

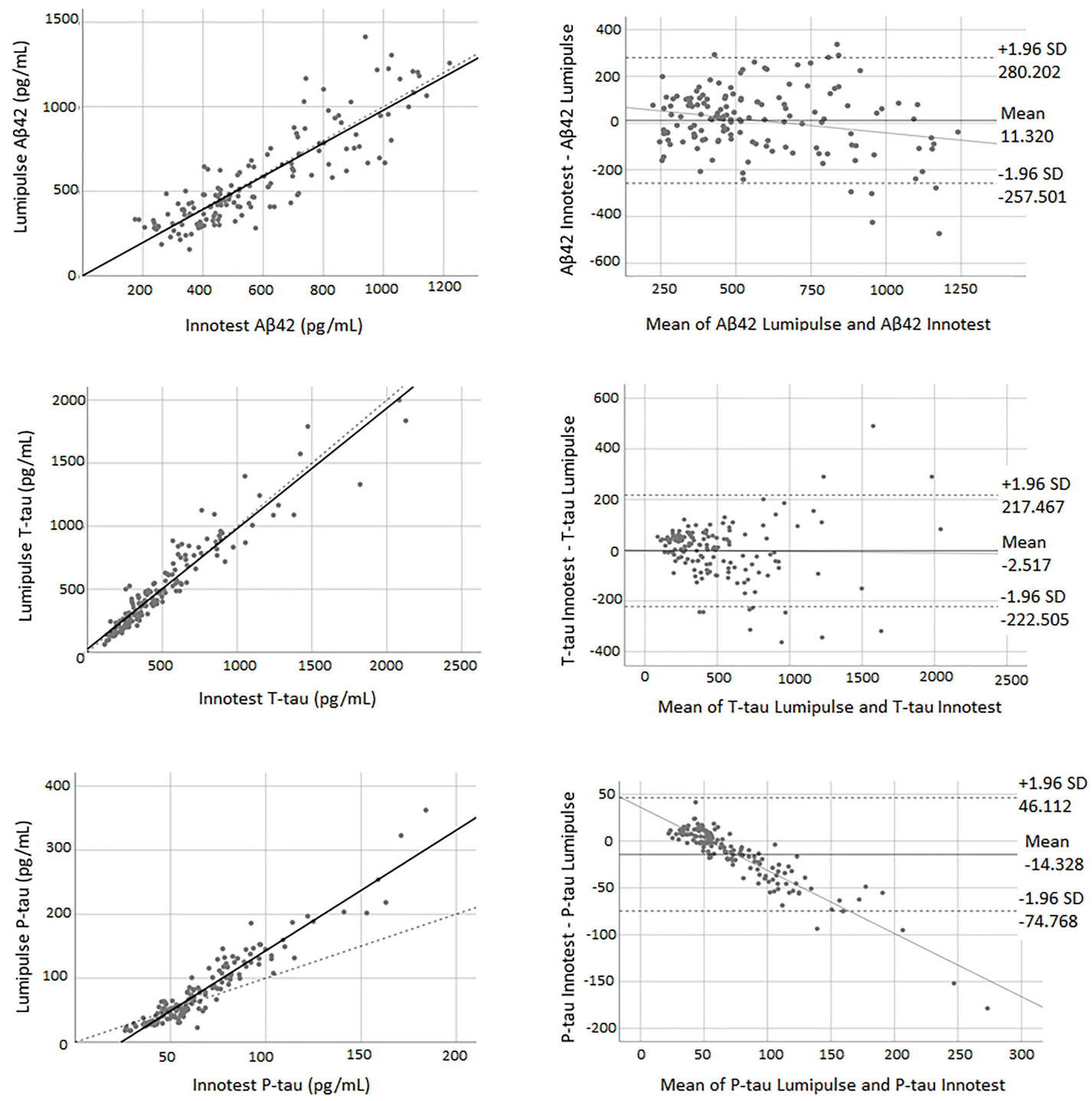

FIGURE 1 | The correlation and Bland-Altman plots for A A42, T-tau, and P-tau measurements obtained by Lumipulse and Innotest ELISA methods ( $n=149$ ). Each point is defined as the measurements of Lumipulse and ELISA assays on the same biological sample. In correlation plots, the solid lines represent the estimated regression line, and the dotted line represents the identity line $(x=y)$. In the Bland-Altman plots, solid lines represent the slope observed.

evaluated, approximately $95 \%$ of measured values were within \pm 1.96 SD of the bias (Figure 2).

\section{Concordance Between Elecsys and Lumipulse Assays}

There was a high correlation between all three biomarkers for both methods. The correlation coefficient between the two methods was 0.94 for A $\beta 42(P<0.0001)$, 0.95 for T-tau $(P<0.0001)$, and 0.96 for $P$-tau $(P<0.0001)$. Figure 3 shows the correlation and Bland-Altman plots for biomarkers quantified by Elecsys and Lumipulse. The $t$-test results indicated that there was weak concordance between all pairs of biomarkers $(P<0.0001)$. For all three biomarkers, the adjustment line (continuous line) (observed slope of 0.59 for A $\beta 42$, $t$-test
$P<0.0001$; observed slope of 2.07 for T-tau, $t$-test $P<0.0001$; and observed slope 3.21 for P-tau, t-test $P<0.0001)$ was significantly separated from the perfect agreement line (dashed line) (Figure 3). The Bland-Altman plot indicated that there was a bias of 243.28 (continuous line) for A $\beta 42$, meaning that Lumipulse quantified $243.28 \mathrm{pg} / \mathrm{mL}$ less $\mathrm{A} \beta 42$ on average than Elecsys. The regression line demonstrated a proportional systemic bias with a positive trend of differences as the magnitude of $\mathrm{A} \beta 42$ increased. For T-tau and P-tau, the biases between the two assays were -210.754 and -54.128 units, respectively. The regression line of the differences indicated a proportional systematic bias for both biomarkers with a negative trend of differences as the magnitude of these biomarkers increased. For all assays evaluated, approximately $95 \%$ of measured values were within $\pm 1.96 \mathrm{SD}$ of the bias (Figure 3). 

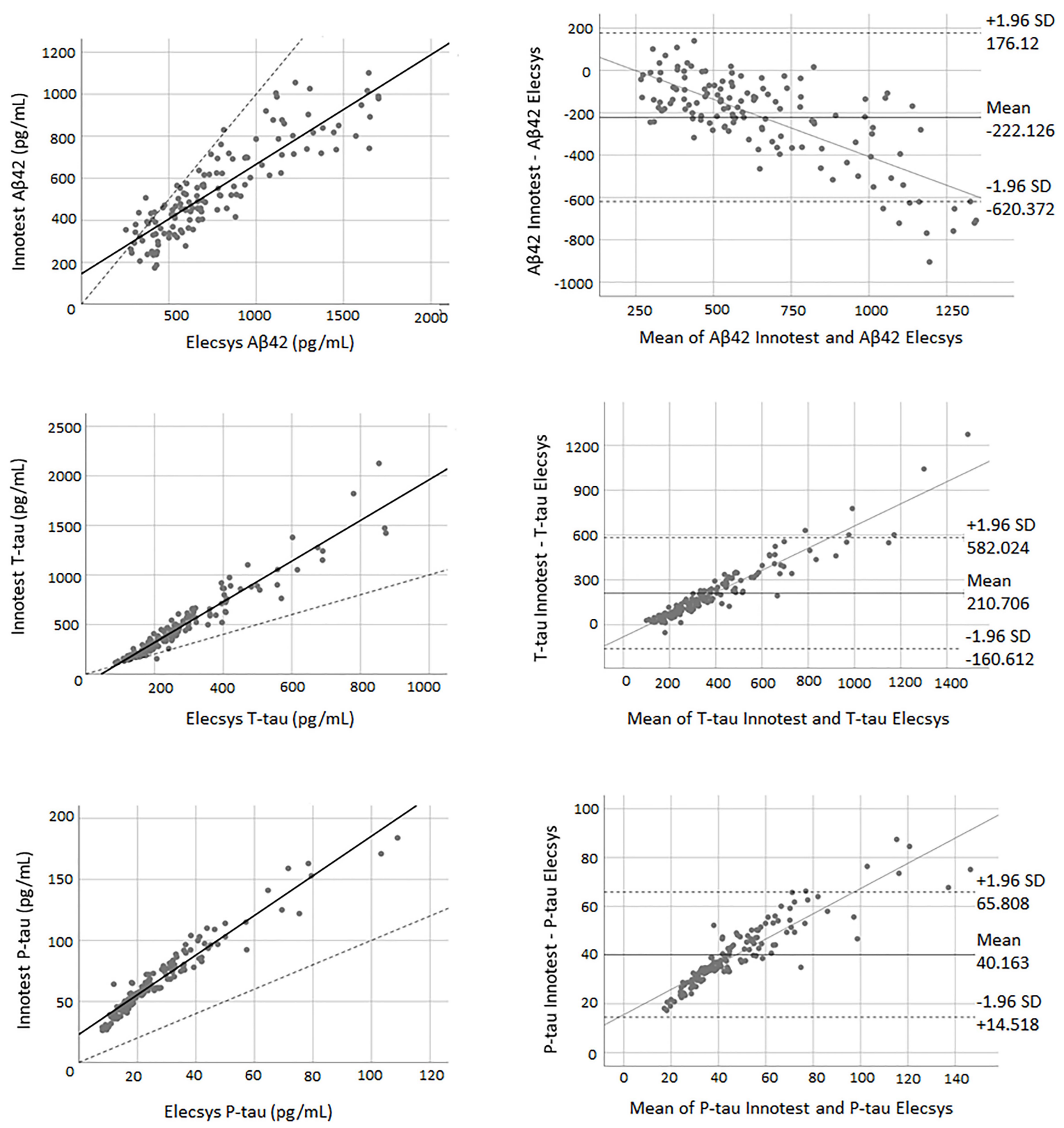

FIGURE 2 | The correlation and Bland-Altman plots for Aß42, T-tau, and P-tau measurements obtained by Elecsys and Innotest methods (for P-tau and T-tau $n=145$; for A $442 n=135$ ). Each point is defined as the measurement of Elecsys and Innotest assays on the same biological sample. In correlation plots, the solid lines represent the estimated regression line, and the dotted line represents the identity line $(x=y)$. In the Bland-Altman plots, solid lines represent the slope observed.

\section{AD Diagnostic Accuracy of the Biomarkers Quantified by Each Method}

Using binary logistic regression, we evaluated the diagnostic accuracy of biomarkers quantified by each assay (clinical diagnosis is generally considered the gold standard). To discriminate $\mathrm{AD}$ from non-AD patients, the combined use of $\mathrm{A} \beta 42$ and P-tau was the best approach for all three assays. The $A \beta 42 / 40$ ratio of Lumipulse also had high discriminating power, comparable with the combined use of $A \beta 42$ and P-tau, to differentiate between the two diagnostic groups (AUC 0.882,
95\% CI 0.785-0.980). Among all three methods, Lumipulse A $\beta 42$ and P-tau had higher discriminating power with an AUC of 0.915 (95\% CI 0.822-1.000). This combination of biomarkers had $91.2 \%$ sensitivity and $76.2 \%$ specificity for a correct classification of diagnostic groups, and their predictive accuracy was estimated to be $85.5 \%$. However, the AUCs were not significantly different between the three methods, as they were assessed using the Hanley and McNeil method $(|z|<1.96)$. However, the sensitivity, specificity and predictive accuracy slightly differed between methods (Table 2). 

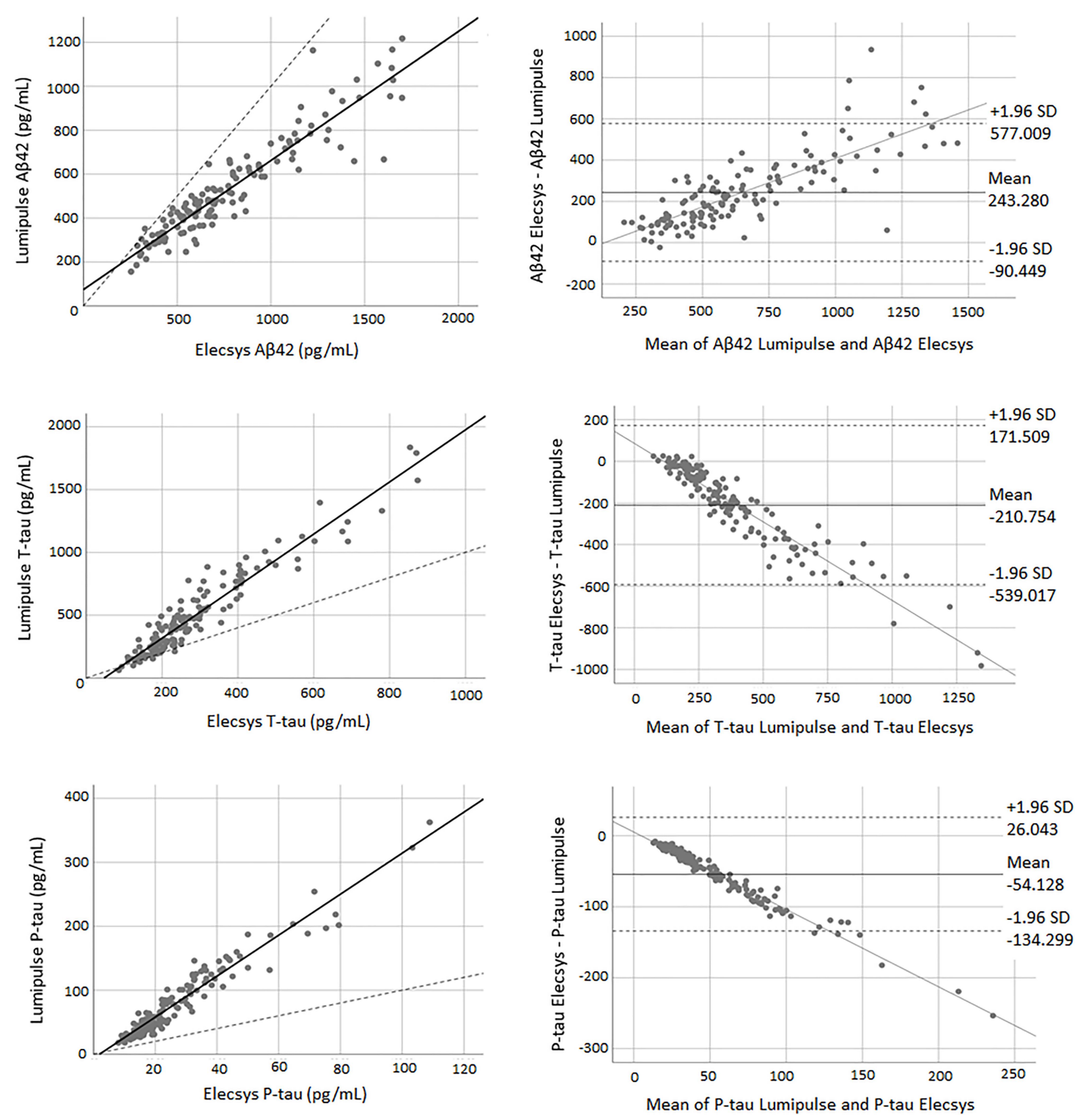

FIGURE 3 | The correlation and Bland-Altman plots for A 42 , T-tau, and P-tau measurements obtained by Lumipulse and Elecsys methods (for P-tau and T-tau $n=145$; for A $442 n=137$ ). Each point is defined as the measurement of Lumipulse and Elecsys assays on the same biological sample. In correlation plots, the solid lines represent the estimated regression line, and the dotted line represents the identity line $(x=y)$. In the Bland-Altman plots, solid lines represent the slope observed.

TABLE 2 | Biomarkers with the best discriminating power between AD and non-AD dementia patients.

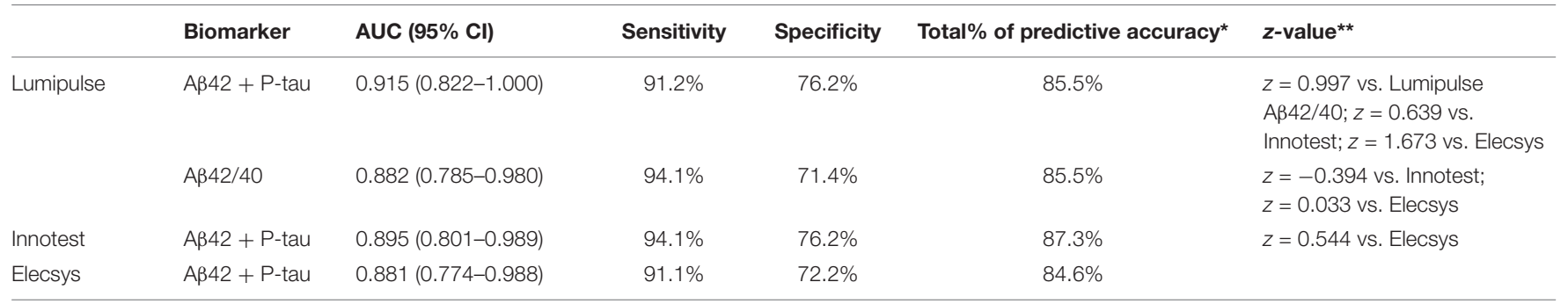

AUC, Area under the curve.

*The percentage of correct classification of $A D+$ correct classification of non-AD/all cases.

${ }^{* *}$ Values of $|z|<1.96$ were taken as evidence that the true ROC areas were not different. 


\section{Diagnostic Accuracy of the AT(N) Classification for Each Method}

The same statistical model was used to evaluate the discriminating power of the $\mathrm{AT}(\mathrm{N})$ classification for each method. We classified our study population into $6 \operatorname{AT}(\mathrm{N})(0$, $1,2,3,4$, and 5) groups based on the results of the three core AD biomarkers (Jack et al., 2018). Biomarkers were grouped into those for $\beta$ amyloid deposition, pathologic tau, and neurodegeneration $[\mathrm{AT}(\mathrm{N})]$. Here, A referred to levels of $\mathrm{A} \beta 42$ $(\mathrm{A} \beta 42 / 40)$ in CSF, $\mathrm{T}$ referred to levels of P-tau in CSF, and $(\mathrm{N})$ referred to levels of T-tau in CSF. We provided two AT(N) classifications for Lumipulse, one based on the results of $A \beta 42$, T-tau and P-tau and the other one based on the A $\beta 42 / 40$, T-tau and P-tau values (Table 3). Patients who were grouped as AT(N) 0 were negative for all three biomarkers. Patients in the AT(N) 1 group were only positive for $\mathrm{A} \beta 42$ or the $\mathrm{A} \beta 42 / 40$ ratio. $\mathrm{AT}(\mathrm{N}) 2$ patients were positive for $A \beta 42$ or the $A \beta 42 / 40$ ratio and P-tau. AT(N) 3 patients had positive results for all three biomarkers. $\mathrm{AT}(\mathrm{N}) 4$ patients were positive for $\mathrm{A} \beta 42$ or the $\mathrm{A} \beta 42 / 40$ ratio and T-tau. Finally, AT(N) 5 patients were negative for $A \beta 42$ or the A $\beta 42 / 40$ ratio but positive for P-tau or T-tau or both biomarkers. For Lumipulse and Elecsys assays, classification was made based on the cut-offs provided by the manufacturers. The cut-offs for the Innotest assay were determined in an independent cohort of patients and controls in our lab. Our results indicated that although $\mathrm{AT}(\mathrm{N})$ classification based on the $\mathrm{A} \beta 42 / 40$ had the best discriminating power to correctly separate $\mathrm{AD}$ patients from non-AD patients with dementia (AUC 0.798; 95\% CI $0.649-0.947)$, there were no significant differences between the four $\mathrm{AT}(\mathrm{N})$ classifications [i.e., Innotest, 2 lumipulse and Elecsys biomarkers based on the AT(N) classifications] with respect to diagnostic accuracy after comparing AUCs with the Hanley and McNeil method $(|z|<1.96)$. However, the sensitivity, specificity, and total percentage of predictive accuracy were different between methods, especially between Lumipulse and Innotest or Elecsys (Table 3). Among the three methods, Lumipulse AT(N)s had the best sensitivity (91.2\%) and total predictive accuracy, while Elecsys $\mathrm{AT}(\mathrm{N})$ had the best specificity $(77.8 \%)$ for discriminating $\mathrm{AD}$ from non-AD dementia patients.

\section{CSF Biomarker Cut-Offs Based on A $\beta 42 / 40$ Ratio Status}

As the $A \beta 42 / 40$ ratio and $A T(N)$ had the best diagnostic accuracy, we selected these variables to serve as references for determining the cut-offs of biomarkers and ratios for Lumipulse and Elecsys. The cut-offs for each biomarker or ratio were established to be values that optimized the concordance with $A \beta 42 / 40$ status as positive/negative. The determined cut-offs in this study and the established cut-offs by Fujirebio and Roche Diagnostics are presented in Table 4. As displayed in Table 4, the cut-offs

TABLE 3 | Diagnostic accuracy of the AT(N) classification for each method.

\begin{tabular}{|c|c|c|c|c|c|c|}
\hline & $\mathrm{AT}(\mathrm{N})$ & AUC $(95 \% \mathrm{Cl})$ & Sensitivity & Specificity & Total\% of predictive accuracy ${ }^{\star}$ & $z$-value ${ }^{\star \star}$ \\
\hline \multirow[t]{2}{*}{ Lumipulse } & A $\beta 42 / 40$, P-tau, (T-tau) & $0.798(0.649-0.947)$ & $91.2 \%$ & $71.4 \%$ & $83.6 \%$ & $\begin{array}{l}z=0.432 \text { vs. Lumipulse } A \beta 42 \\
\text { P-tau, (T-tau); } 0.288 \text { vs. } \\
\text { Innotest; } z=0.307 \text { vs. Elecsys }\end{array}$ \\
\hline & A $\beta 42$, P-tau, (T-tau) & $0.778(0.617-0.939)$ & $91.2 \%$ & $71.4 \%$ & $83.6 \%$ & $\begin{array}{l}z=-0.076 \text { vs. Innotest; } \\
z=-0.034 \text { vs. Elecsys }\end{array}$ \\
\hline Innotest & A $\beta 42$, P-tau, (T-tau) & $0.783(0.627-0.938)$ & $79.4 \%$ & $76.2 \%$ & $78.2 \%$ & $z=-0.052$ vs. Elecsys \\
\hline Elecsys & A $\beta 42$, P-tau, (T-tau) & $0.780(0.624-0.937)$ & $67.6 \%$ & $77.8 \%$ & $67.3 \%$ & \\
\hline
\end{tabular}

AUC, Area under the curve.

*The percentage of correct classification of $A D+$ correct classification of non-AD/all cases.

${ }^{*}$ Values of $|z|<1.96$ were taken as evidence that the true ROC areas were not different.

TABLE 4 | Cut-offs of CSF biomarkers that yielded maximum Youden index versus A $\beta 42 / A \beta 40$ ratio status in the receiver operating characteristics analysis.

\begin{tabular}{|c|c|c|c|c|c|c|c|c|}
\hline & & AUC (95\% Cl) & PPA & NPA & Max Youden index & Cut-off & OPA & Manufacturer cutoffs \\
\hline \multirow[t]{6}{*}{ Lumipulse } & T-tau & $0.860(0.791-0.930)$ & $72.5 \%$ & $89.4 \%$ & $61.9 \%$ & $\geq 399$ & $77.9 \%$ & $>400$ \\
\hline & P-tau & $0.925(0.884-0.967)$ & $86.3 \%$ & $85.1 \%$ & $71.4 \%$ & $\geq 51$ & $85.9 \%$ & $>56.5$ \\
\hline & $\mathrm{A} \beta 42$ & $0.923(0.878-0.967)$ & $82.4 \%$ & $89.4 \%$ & $71.7 \%$ & $\leq 563$ & $84.6 \%$ & $<600$ \\
\hline & $\mathrm{A} \beta 42 / 40$ & $1.000(1.000-1.000)$ & $100 \%$ & $100 \%$ & $100 \%$ & $\leq 0.070$ & $100 \%$ & $<0.069$ \\
\hline & P-tau/A $\beta 42$ & $0.992(0.984-1.000)$ & $95.1 \%$ & $97.9 \%$ & $93.0 \%$ & $\geq 0.082$ & $95.9 \%$ & - \\
\hline & T-tau/A $\beta 42$ & $0.956(0.906-1.000)$ & $96.1 \%$ & $93.6 \%$ & $89.7 \%$ & $\geq 0.517$ & $95.3 \%$ & - \\
\hline \multirow[t]{5}{*}{ Elecsys } & T-tau & $0.812(0.739-0.885)$ & $58.0 \%$ & $95.6 \%$ & $53.6 \%$ & $\geq 268.15$ & $69.7 \%$ & $>300$ \\
\hline & P-tau & $0.867(0.811-0.923)$ & $70.0 \%$ & $95.6 \%$ & $65.6 \%$ & $\geq 22.175$ & $78.0 \%$ & $>27$ \\
\hline & $\mathrm{A} \beta 42$ & $0.904(0.840-0.967)$ & $93.0 \%$ & $78.4 \%$ & $71.4 \%$ & $\leq 939.150$ & $89.1 \%$ & $\leq 1000$ \\
\hline & P-tau/A $\beta 42$ & $0.994(0.986-1.000)$ & $96.0 \%$ & $97.3 \%$ & $93.3 \%$ & $\geq 0.023$ & $96.4 \%$ & $>0.024$ \\
\hline & T-tau/Aß42 & $0.974(0.932-1.000)$ & $96.0 \%$ & $94.6 \%$ & $90.6 \%$ & $\geq 0.26$ & $95.6 \%$ & $>0.28$ \\
\hline
\end{tabular}

AUC, Area under the curve; PPA, Positive percent agreement with A $42 / 40$ status; NPA, Negative percent agreement with A $42 / 40$ status; Max Youden index, $(P P A+N P A-1) ; O P A$, Overall percent agreement. 


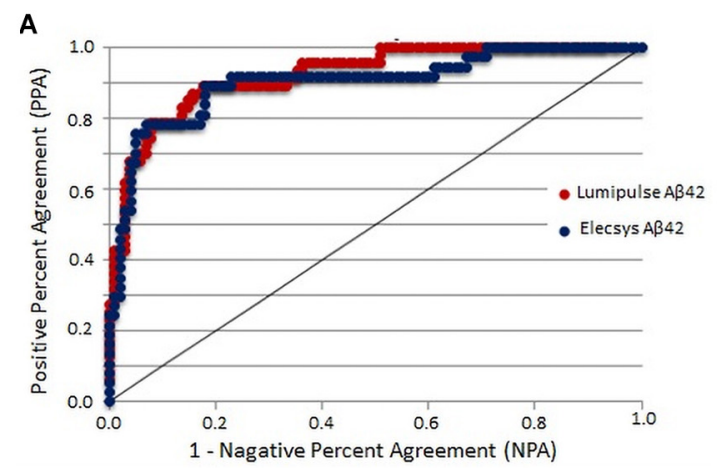

C

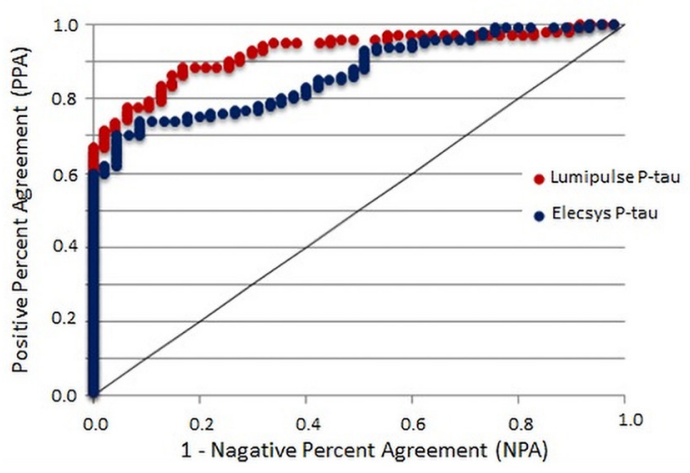

E

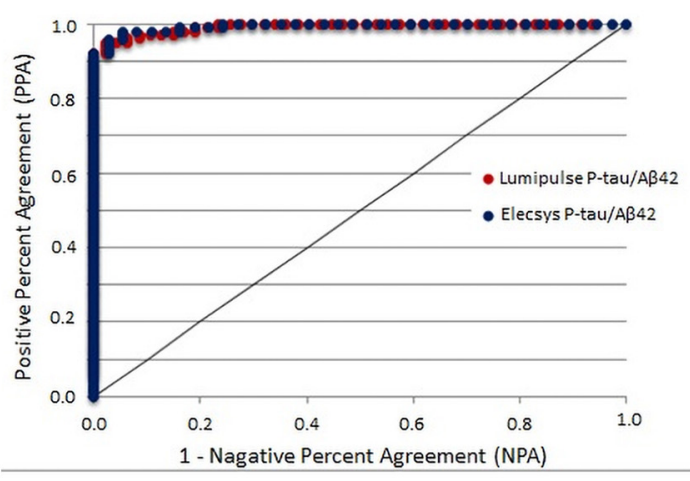

B

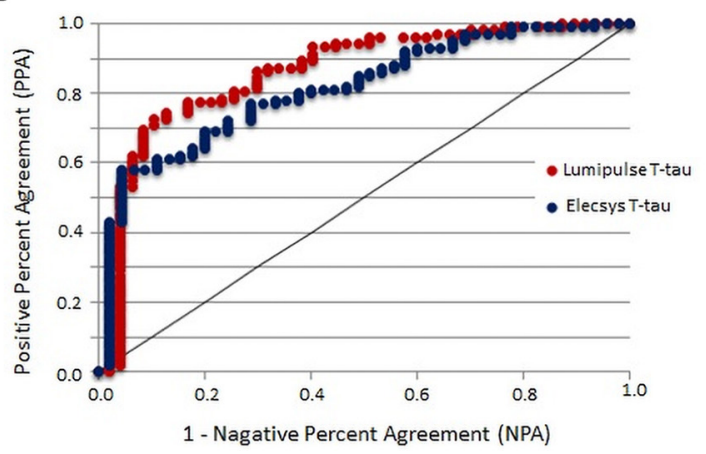

D

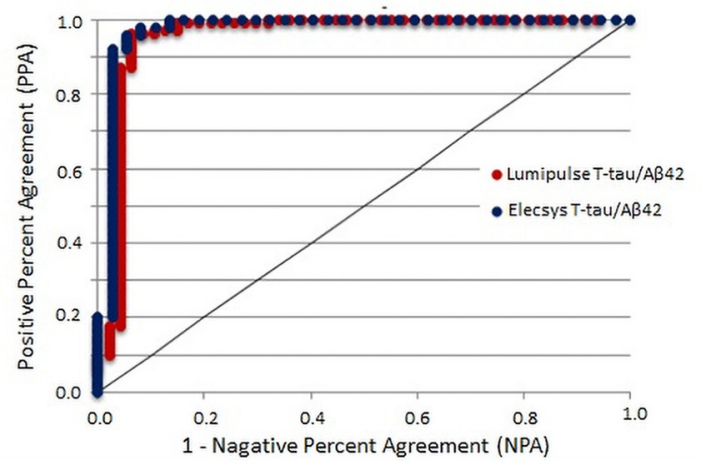

FIGURE 4 | CSF biomarkers that yielded the maximum Youden index versus A $\beta 42 / A \beta 40$ ratio status in the receiver operating characteristics analysis.

determined according to concordance with the $A \beta 42 / 40$ ratio were comparable with the manufacturer cut-offs. For Lumipulse and Elecsys biomarkers and ratios, the AUC for discriminating $\mathrm{A} \beta 42 / 40$ status was greater than 0.8 . To discriminate between $\mathrm{A} \beta 42 / 40$ positivity/negativity status among Lumipulse assays, the $\mathrm{A} \beta 42 / 40$ AUC was 100 , as it was used for calculating the cutoffs. However, the P-tau/A $\beta 42$ and T-tau/A $\beta 42$ ratios had a high discriminating accuracy (AUC 0.922, OPA 95.9\% and AUC 0.956, OPA $95.3 \%$, respectively) at the cut-off values of $\geq 0.082$ and $\geq 0.517$, respectively. Among Elecsys markers, the P-tau/A $\beta 42$ and $\mathrm{T}$-tau/A $\beta 42$ ratios had superior discriminating power (AUC
0.994, OPA 96.4\% and AUC 0.974, OPA 95.6\%, respectively) at the cut-off values of $\geq 0.023$ and $\geq 0.26$, respectively. In fact, the Elecsys P-tau/A $\beta 42$ was the best in discriminating patients based on A $\beta 42 / 40$ status (Table 4 and Figure 4).

In addition, we determined the cut-offs for biomarkers and ratios of Lumipulse and Elecsys based on the $A \beta 42$ status of Innotest (Supplementary Table 1). Among Lumipulse assays, A $\beta 42$ had the highest AUC $(0.955,95 \%$ CI 0.924-0.986) followed by P-tau/A $\beta 42$ (AUC 0.933, 95\% CI 0.891-0.976) and $A \beta 42 / 40$ (AUC 0.931, 95\% CI 0.892-0.970). For the Elecsys assay, A $\beta 42$ had the highest AUC (0.974, 95\% CI 0.954-0.995), followed by 
P-tau/A 42 (AUC 0.936, 95\% CI 0.894-0.978) and T-tau/A $\beta 42$ (AUC 0.920, 95\% CI 0.868-0.971).

Furthermore, all statistical analysis was performed for the study cohort separated by sex (Supplementary Figures 1-8 and Supplementary Tables 2-7). We found a high correlation between the three methods for both male and female subjects, although there were systematic differences between biomarker values measured by each method (Supplementary Figures 1-6). Interestingly, the cut-offs of CSF biomarkers for male subjects was lower than those of manufacturer, while in the case of female subjects these cut-offs were comparable with corresponding manufacturer's cut-offs (Supplementary Tables 6, 7).

\section{DISCUSSION}

In this study, we evaluated the concordance between three different methods for measurement of AD CSF biomarkersInnotest ELISA, Elecsys and Lumipulse platforms-in a cohort of patients with $\mathrm{AD}, \mathrm{MCI}$, and non-AD dementias. We also evaluated the diagnostic accuracy of biomarkers and their ratios measured by each method. Furthermore, we determined cut-offs for CSF biomarkers of AD (Aß42, T-tau, and P-tau) and their ratios measured on the fully automated Lumipulse and Elecsys to optimize their concordance with $\mathrm{A} \beta 42 / 40$ status.

Although there was a high correlation between all three assays, our results showed that there was a lack of consistency between the three methods, except for $A \beta 42$ and T-tau of Lumipulse and Innotest. Because the antibodies used for the Lumipulse assays were produced by the same manufacturer as Innotest ELISA (Fujirebio), the similar specificity of the antibodies between the two methods may partly explain the concordance we observed between $\mathrm{A} \beta 42$ and $\mathrm{T}$-tau values between these two methods. Our results were consistent with previous studies that had found a systematic bias between the measurements of biomarkers by Lumipulse and Innotest (Bayart et al., 2019) and by Elecsys and Innotest (Willemse et al., 2018). The lack of concordance between Elecsys, Lumipulse, and Innotest assays may be attributed to the differences that exist between these methods. First, they have different recommended preanalytical procedures that can affect the measured concentration of CSF biomarkers. Among these three biomarkers, A $\beta 42$ is known to be more sensitive to pre-analytical conditions. Second, these methods use different measurement technologies (ECLIA, CLEIA, and ELISA, respectively), which may affect the detectable concentration. Third, the antibodies that were produced and applied in the AD CSF assays by Roche Diagnostics and Fujirebio Diagnostics may have different specificities. Finally, although both Elecsys and Lumipulse have been standardized for A $\beta 42$, the material used for standardization differed between methods (Bittner et al., 2016; Kuhlmann et al., 2017).

We examined the ability of $\mathrm{A} \beta 42$, T-tau, P-tau and their ratios to discriminate $\mathrm{AD}$ patients from patients with non$\mathrm{AD}$ dementias. $\mathrm{A} \beta 42$ and P-tau combined were both the best biomarkers for discriminating between the two diagnostic groups. Both biomarkers were specific to $\mathrm{AD}$; therefore, it was not surprising that their combination had a high discriminating power for diagnosing $\mathrm{AD}$ patients. However, abnormal concentrations of T-tau in CSF, which underlies neurodegeneration, is not specific to $\mathrm{AD}$ and occurs in non$\mathrm{AD}$ dementias or in non-AD elderly persons with comorbidities (Kovacs et al., 2013). Evaluation of differences in AUC revealed that there were no significant differences in the discriminating power of $\mathrm{A} \beta 42+\mathrm{P}$-tau measured by each method. However, the Innotest $\mathrm{A} \beta 42+\mathrm{P}$-tau had a better sensitivity (94\%), specificity (76\%), and predictive accuracy (87\%). The $A \beta 42 / 40$ ratio also had a high discriminating power for differentiating between patients with $\mathrm{AD}$ and non- $\mathrm{AD}$ dementias. Consistent with our results, Shoji et al. (1998) and Lewczuk et al. (2004) previously suggested that the $A \beta 42 / 40$ ratio is superior to the concentration of $A \beta 42$ alone for discriminating $\mathrm{AD}$ patients.

We also assessed the discriminating power of $\mathrm{AT}(\mathrm{N})$ groups that were generated by the results of CSF $A \beta 42(A \beta 42 / 40)$, P-tau and T-tau for each method. The AT(N) classification was proposed by the NIAA research framework (Jack et al., 2018) and gives a biological rather than a clinical definition of $\mathrm{AD}$. We found that the use of $\mathrm{A} \beta 42 / 40$ instead of $\mathrm{A} \beta 42$ in AT(N) improved the classification accuracy (AUC 0.798, 95\% CI $0.649-0.947$ vs. AUC $0.778,95 \%$ CI $0.617-0.939)$, However, the sensitivity, specificity and predictive accuracy was the same for both $\mathrm{AT}(\mathrm{N})$ s. Among all four $\mathrm{AT}(\mathrm{N})$ classifications, Elecsys $\mathrm{AT}(\mathrm{N})$ had the highest specificity. In fact, Elecsys AT(N) had better specificity than sensitivity in discriminating the two diagnostic groups. The preference for higher sensitivity or specificity depends on the purpose of different investigation scenarios. For example, for screening purposes, higher sensitivity is always preferable; however, high specificity might be preferable for the selection of patients for clinical trials. These results should be interpreted with caution because of the small population size of both of the diagnostic groups in our study.

Finally, we defined the CSF cut-offs for both Lumipulse and Elecsys assays based on the Lumipulse $A \beta 42 / 40$ status because of its high diagnostic accuracy in our study, its high stability with respect to pre-analytical variations (Lewczuk et al., 2006; Willemse et al., 2018) and the fact that the ratio probably accounts for inter-individual variability in overall $A \beta$ production and CSF turnover (Janelidze et al., 2017). Given that Innotest assays are among some of the most commonly used methods for the detection of AD CSF biomarkers, we also provided the cut-offs for both Lumipulse and Elecsys assays based on the Innotest A $\beta 42$ status.

In previous studies, amyloid PET visual read (Schindler et al., 2018; Alcolea et al., 2019) or diagnostic accuracy (Bayart et al., 2019) have been used for the determination of AD CSF biomarkers cut-offs for fully automated methods and their ratios. Our results indicated that the cut-offs based on the $A \beta 42 / 40$ ratio had a close similarity to the cut-offs established by each manufacturer; therefore, the $A \beta 42 / 40$ ratio is a robust variable that can differentiate $\mathrm{AD}$ from non-AD individuals. Based on our results for both the Lumipulse and the Elecsys methods, $\mathrm{P}$-tau/A $\beta 42$ and $\mathrm{T}-\mathrm{tau} / \mathrm{A} \beta 42$ performed better together than each biomarker alone in discriminating $\mathrm{A} \beta 42 / \mathrm{A} \beta 40 \pm$ status. This result is consistent with the results of previous studies where P-tau/A $\beta 42$ (Alcolea et al., 2019) or T-tau/A $\beta 42$ 
(Bayart et al., 2019) demonstrated superior performance in discriminating the diagnostic groups or amyloid PET status compared with individual biomarkers (Schindler et al., 2018).

Some limitations of this study require consideration. First, our study population lacked health control individuals. The majority of the population consisted of MCI subjects $(n=94)$ with a short follow-up time; for this reason, we decided to eliminate patients in some analyses and retain a small number of $\mathrm{AD}$ $(n=34)$ and non-AD demented patients $(n=21)$ when evaluating the diagnostic accuracy of biomarkers. Second, instead of using an independent method, we used the $A \beta 42 / A \beta 40$ ratio status or $A \beta 42$ status to determine the biomarker cut-offs, and this may have led to the overfitting of the results. Third, A $\beta 40$ cannot be measured by Elecsys or ELISA, so, the comparison was incomplete. Other limitation is that 17 patients were excluded of the analyses because they had $A \beta 42$ values above the upper limit of detection (1700 pg/ml) for Elecsys.

The main strength of our study is that we compared, for the first time, the clinical and analytical performance of fully automated Elecsys and Lumipulse platforms together in the same cohort of patients. In addition, our study population consisted of a real population of patients who attended a memory clinic and, therefore, provided a more realistic application of biomarkers in daily clinical practice.

\section{CONCLUSION}

In conclusion, both Lumipulse and Elecsys methods had a high correlation with each other and with Innotest ELISA. The presence of systematic bias between biomarkers measured by each method was expected as there were various pre-analytical and analytical differences between methods. For both Lumipulse and Elecsys methods, ratios had a better analytical performance compared with individual biomarkers, and the $\mathrm{A} \beta 42 / \mathrm{A} \beta 40$ ratio had a high concordance with the diagnostic accuracy of AD. Because the calibrators were adjusted with reference samples in both automated platforms, it was expected that these platforms would reduce intra- and inter-laboratory variations and enhance reproducibility.

\section{AUTHOR'S NOTE}

Considering the importance of study of cerebrospinal fluid biomarkers in mild cognitive impairment and Alzheimer's disease, we aimed to investigate the concordance between core AD biomarkers measured in CSF using Innotest, Lumipulse and Elecsys methods. We observed that both,

\section{REFERENCES}

Albert, M. S., DeKosky, S. T., Dickson, D., Dubois, B., Feldman, H. H., Fox, N. C., et al. (2011). The diagnosis of mild cognitive impairment due to Alzheimer's disease: recommendations from the National Institute on AgingAlzheimer's Association workgroups on diagnostic guidelines for Alzheimer's disease. Alzheimers Dement. 7, 270-279.
Lumipulse and Elecsys methods had a high correlation with each other and with Innotest ELISA. The presence of systematic bias between biomarkers measured by each method was expected as there are various pre-analytical and analytical differences between methods. For both Lumipulse and Elecsys methods, ratios had a better analytical performance compared with individual biomarkers. The Lumipulse and Elecsys CSF AD assays showed high analytical and clinical performances so their use is recommended for the measurement of CSF AD biomarkers compared with unstandardized manual methods.

\section{DATA AVAILABILITY STATEMENT}

The raw data supporting the conclusions of this article will be made available by the authors, without undue reservation.

\section{ETHICS STATEMENT}

The studies involving human participants were reviewed and approved by the Comite Etica Hospital Arnau Vilanova Lleida. The patients/participants provided their written informed consent to participate in this study.

\section{AUTHOR CONTRIBUTIONS}

FD, RL-O, and GP-R designed the study, analyzed the data, interpreted the data, and wrote the manuscript. IR-L, AA, $\mathrm{RH}, \mathrm{NT}$, and MR-J collected the data. All authors revised the manuscript and approved it for submission.

\section{FUNDING}

This study was supported by the Generalitat of Catalonia, Department of Health (PERIS 2019 SLT008/18/00050 to GP-R). IRBLleida is a CERCA Program/Generalitat of Catalonia. FD was supported by Agency for Management of University and Research Grants and European Social Fund (FI_B100153).

\section{SUPPLEMENTARY MATERIAL}

The Supplementary Material for this article can be found online at: https://www.frontiersin.org/articles/10.3389/fnagi. 2021.604119/full\#supplementary-material

Alcolea, D., Pegueroles, J., Muñoz, L., Camacho, V., López-Mora, D., FernándezLeón, A., et al. (2019). Agreement of amyloid PET and CSF biomarkers for Alzheimer's disease on lumipulse. Ann. Clin. Transl. Neurol. 6, 1815-1824. doi: $10.1002 /$ acn 3.50873

Bayart, J. L., Hanseeuw, B., Ivanoiu, A., and van Pesch, V. (2019). Analytical and clinical performances of the automated Lumipulse cerebrospinal fluid $\mathrm{A} \beta 42$ and T-Tau assays for Alzheimer's disease 
diagnosis. J. Neurol. 266, 2304-2311. doi: 10.1007/s00415-019-09 418-6

Bittner, T., Zetterberg, H., Teunissen, C. E., Ostlund, R. E. Jr., Militello, M., Andreasson, U., et al. (2016). Technical performance of a novel, fully automated electrochemiluminescence immunoassay for the quantitation of $\beta$-amyloid (142) in human cerebrospinal fluid. Alzheimers Dement. 12, 517-526. doi: 10. 1016/j.jalz.2015.09.009

Gorno-Tempini, M. L., Hillis, A. E., Weintraub, S., Kertesz, A., Mendez, M., Cappa, S. F., et al. (2011). Classification of primary progressive aphasia and its variants. Neurology 76, 1006-1014.

Hanley, J., and McNeil, B. (1982). The meaning and use of the area under a Receiver Operatin Characteristic (ROC) curve. Radiology 143, 29-36. doi: 10.1148/radiology.143.1.7063747

Hansson, O., Seibylc, J., Stomrud, E., Zetterberg, H., Trojanowskih, J., Bittneri, T., et al. (2018). CSF biomarkers of Alzheimer's disease concord with amyloid- $\beta$ PET and predict clinical progression: a study of fully automated immunoassays in BioFINDER and ADNI cohorts. Alzheimers Dement. 14, 1470-1481. doi: 10.1016/j.jalz.2018.01.010

Jack, C. Jr., Bennett, D., Blennow, K., Carrillo, M., Dunn, B., Haebeerlein, S., et al. (2018). NIA-AA research framework: toward a biological definition of Alzheimer's disease. Alzheimers Dement. 14, 535-562. doi: 10.1016/j.jalz.2018. 02.018

Janelidze, S., Pannee, J., Mikulskis, A., Chiao, P., Zetterberg, H., Blennow, K., et al. (2017). Concordance between different amyloid immunoassays and visual amyloid positron emission tomographic assessment. JAMA Neurol. 74, $1492-$ 1501. doi: 10.1001/jamaneurol.2017.2814

Kaplow, J., Vandijck, M., Gray, J., Kanekiyo, M., Huyck, E., Traynham, C. J., et al. (2020). Concordance of Lumipulse cerebrospinal fluid t-tau/A $\beta 42$ ratio with amyloid PET status. Alzheimers Dement. 16, 144-152. doi: 10.1002/alz.12000

Kollhoff, A. L., Howell, J. C., and Hu, W. T. (2018). Automation vs. experience: measuring Alzheimer's beta-amyloid 1-42 peptide in the CSF. Front. Aging Neurosci. 10:253. doi: 10.3389/fnagi.2018.00253

Kovacs, G. G., Milenkovic, I., Wohrer, A., Hoftberger, R., Gelpi, E., Haberler, C., et al. (2013). Non-Alzheimer neurodegenerative pathologies and their combinations are more frequent than commonly believed in the elderly brain: a community-based autopsy series. Acta Neuropathol. 126, 365-384. doi: 10. 1007/s00401-013-1157-y

Kuhlmann, J., Andreasson, U., Pannee, J., Bjerke, M., Portelius, E., Leinenbach, A., et al. (2017). CSF Ab 1-42 -an excellent but complicated Alzheimer's biomarker - a route to standardisation. Clin. Chim. Acta 467, 27-33. doi: 10.1016/j.cca.2016.05.014

Leinenbach, A., Pannee, J., Dulffer, T., Huber, A., Bittner, T., Andreasson, U., et al. (2014). Mass spectrometry-based candidate reference measurement procedure for quantification of amyloid-beta in cerebrospinal fluid. Clin. Chem. 60, 987-994. doi: 10.1373/clinchem.2013.220392

Lewczuk, P., Beck, G., Esselmann, H., Bruckmoser, R., Zimmermann, R., Fiszer, M., et al. (2006). Effect of sample collection tubes on cerebrospinal fluid concentrations of tau proteins and amyloid beta peptides. Clin. Chem. 52, 332-334. doi: 10.1373/clinchem.2005.058776

Lewczuk, P., Esselmann, H., Otto, M., Maler, J. M., Henkel, A. W., Henkel, M. K., et al. (2004). Neurochemical diagnosis of Alzheimer's dementia by
CSF Abeta42, Abeta42/Abeta40 ratio and total tau. Neurobiol. Aging 25, 273-281.

McKeith, I. G., Boeve, B. F., Dickson, D. W., Halliday, G., Taylor, J. P., Weintraub, D., et al. (2017). Diagnosis and management of dementia with Lewy bodies: fourth consensus report of the DLB consortium. Neurology 89, 88-100.

McKhann, G. M., Knopman, D. S., Chertkow, H., Hyman, B. T., Jack, C. R. Jr., Kawas, C. H., et al. (2011). The diagnosis of dementia due to Alzheimer's disease: recommendations from the National Institute on Aging Alzheimer's Association workgroups on diagnostic guidelines for Alzheimer's disease. Alzheimers Dement. 7, 263-269.

Rascovsky, K., Hodges, J. R., Knopman, D., Mendez, M., Kramer, J. H., van Swieten, J., et al. (2011). Sensitivity of revised diagnostic criteria for the behavioural variant of frontotemporal dementia. Brain 134, 24562477.

Schindler, S., Graya, J., Gordona, B., Xionga, C., Batrla-Utermannf, R., Quan, M., et al. (2018). Cerebrospinal fluid biomarkers measured by Elecsys§assays compared to amyloid imaging. Alzheimers Dement. 14, 1460-1469. doi: 10. 1016/j.jalz.2018.01.013

Serrano-Pozo, A., Frosch, M. P., Masliah, E., and Hyman, B. T. (2011). Neuropathological alterations in Alzheimer disease. Cold Spring Harb. Perspect. Med. 1:a006189. doi: 10.1101/cshperspect.a006189

Shoji, M., Matsubara, E., Kanai, M., Watanabe, M., Nakamura, T., Tomidokoro, Y., et al. (1998). Combination assay of CSF tau, A beta 1-40 and A beta 1-42(43) as a biochemical marker of Alzheimer's disease. J. Neurol. Sci. 158, 134-140. doi: 10.1159/000078534

Sperling, R. A., Aisen, P. S., Beckett, L. A., Bennett, D. A., Craft, S., Fagan, A. M., et al. (2011). Toward defining the preclinical stages of Alzheimer's disease: recommendations from the National Institute on Aging Alzheimer's Association workgroups on diagnostic guidelines for Alzheimer's disease. Alzheimers Dement. 7, 280-292.

Willemse, E., van Maurikb, I., Tijmsb, B., Bouwmanb, F., Franked, A., Hubeeke, I., et al. (2018). Diagnostic performance of Elecsys immunoassays for cerebrospinal fluid Alzheimer's disease biomarkers in a nonacademic, multicenter memory clinic cohort: the ABIDE project. Alzheimers Dement. 10, 563-572. doi: 10.1016/j.dadm.2018.08.006

Zecca, C., Brescia, V., Piccininni, M., Capozzo, R., Barone, R., Barulli, M. R., et al. (2019). Comparative evaluation of two immunoassays for cerebrospinal fluid $\beta$ Amyloid1-42 measurement. Clin. Chim. Acta 493, 107-111. doi: 10.1016/j.cca. 2019.02.033

Conflict of Interest: The authors declare that the research was conducted in the absence of any commercial or financial relationships that could be construed as a potential conflict of interest.

Copyright (C) 2021 Dakterzada, López-Ortega, Arias, Riba-Llena, Ruiz-Julián, Huerto, Tahan and Piñol-Ripoll. This is an open-access article distributed under the terms of the Creative Commons Attribution License (CC BY). The use, distribution or reproduction in other forums is permitted, provided the original author(s) and the copyright owner(s) are credited and that the original publication in this journal is cited, in accordance with accepted academic practice. No use, distribution or reproduction is permitted which does not comply with these terms. 